\title{
The Design of Omni-directional Mobile Platform based on the Internet of Things Technology
}

\author{
Shan Gao ${ }^{1}$, Dehui Fan ${ }^{1}$,Lei Zhang ${ }^{2}$ \\ ${ }^{1}$ Qingdao Technical College, Qingdao, The China \\ ${ }^{2}$ Ocean University of China, Qingdao, The China
}

KEYWORD: Robot; Internet of things; Fingerprint localization algorithm

ABSTRACT: This paper provides a design of Omni-directional mobile platform based on Internet of things technology. The platform realized the all-round wheel structure designs, which can flexible complete mobile operation, and have good adaptability to the environment. At the same time, the platform combined the Internet of things technology and robot technology, realized intelligent obstacle avoidance, indoor positioning, data relay and other functions, which had good ability of data processing and expansibility. Through practical verification, this platform can realize human-machine interaction and reliable operation, which has a broad application prospect.

\section{Introduction}

With the development of science and technology, the robot has broad application prospects in scientific exploration, public service, home entertainment, etc. ${ }^{[1]}$ The rapid development of modern electronic technology, Internet technology, automatic control technology, and the deepening of human exploring in various fields, also put forward many new requirements to the robot design. Modern mobile robot research began in the middle of the 20th century, mainly concentrated in a few developed countries such as America, Europe and Japan. Because of mobile robot technology focus on the advanced achievements in multiple disciplines of computer vision, pattern recognition, multisensor fusion, artificial intelligence, communication, virtual reality, etc. and its military, civilian and application prospect in the field of scientific research, it has attracted the attention of governments and big companies. ${ }^{[2]}$

This paper mainly developed an Omni-directional mobile platform based on Internet of things technology, which used the characteristics of the Omni-directional wheel, overcame disadvantages of traditional motion mechanism such as bearing and the obstacle-surmounting performance defects, and also solved the constraints of turning radius, lateral and complex operation and so on. At the same time, the platform used the Internet of things and the advantages of multi-sensor technology, to intelligently collect the field data, automatically connect to the Internet and positioning, and improve the level of intelligent products. ${ }^{[3][4]}$ The system allows the operator by way of Internet, GPRS of mobile devices to query data and transport operations commands. System overall structure is shown in figure 1.

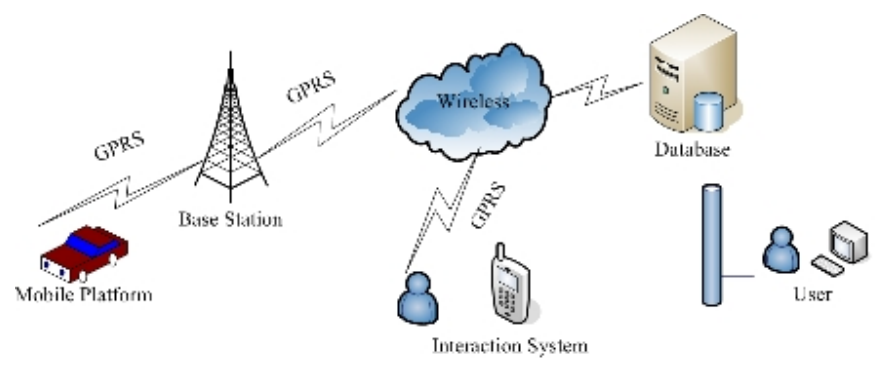

Figure 1. System overall structure.

\section{Control Process}

System control is divided into three parts: database management system, the human-computer interaction system, mobile platform control system. 
(1) Database management system: Implements the query of sensing information and running condition statistics operations to the mobile platform management. The storage depth of this system is three years, which uses day as a unit and displayed all relevant information about the mobile platform on page. Operators can fully understand the working state history information of the mobile platform through database management system which supports wireless and online upgrade function and allows the update operation.

(2) Human-computer interaction system: Software platform for users is based on the android operating system, which realizes flexible instruction and information query function in APP. The system obtains the data of the mobile platform through GPRS ways and communicates with the mobile platform control system through efficient Internet control protocol. The human-computer interaction system and mobile work platform uses $\mathrm{C} / \mathrm{S}$ mode, the former is set as the host. Firstly, the host creates a service, and then accepts the connection application to establish a network connection. Communication command frame format is as shown below:

Table 1. Communication command frame format.

\begin{tabular}{lccc}
\hline & \multicolumn{2}{c}{ Function Frame Byte } \\
ID:23-20 & ID:19-16 & ID:15-8 & ID:7-0 \\
SrcMACID & DestMACID & FuncID & SourceID \\
\hline \multicolumn{4}{c}{} \\
\hline Byte3 & Data Frame Byte \\
CheckFlag & LengthFlag & Byte1 & Byte0 \\
\hline
\end{tabular}

(3) Mobile platform control system: Is responsible for completing the underlying motion control task, and uploads the sensing information and status information, as well as receives other system control commands. The system selects the STM32 microcontroller as the main processing chip, uses IAR for ARM software development environment, and adopts $C$ language in software design. Mobile platform control system is the core system which directly determines the functional characteristics and movement ability of the mobile platform. Functional units mainly include: obstacle avoidance circuit unit, communication unit, monitoring unit, positioning unit, wheel control unit, etc. Structure is as shown in the figure below:

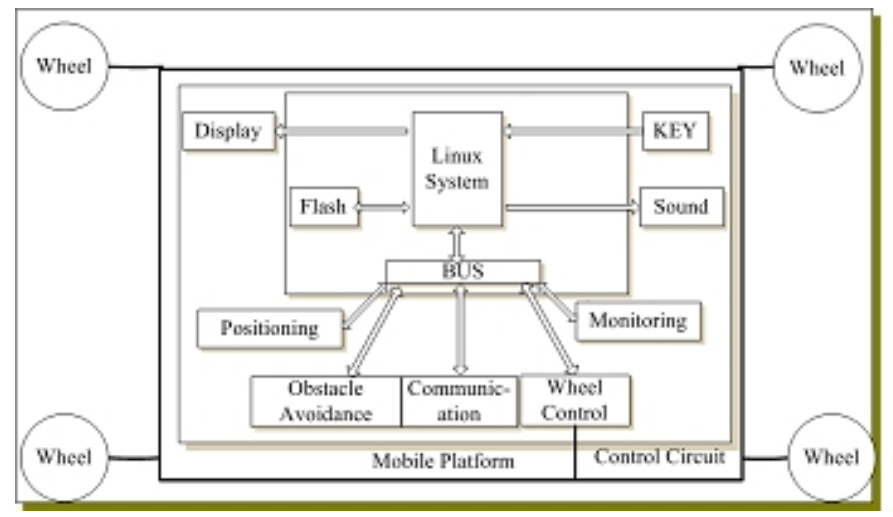

Figure 2. Mobile platform control system structure.

Among them, the obstacle avoidance circuit unit uses ultrasonic wave as the carrier, limits the precision to $1 \mathrm{~cm}$, and measurement range is $3 \mathrm{~cm}$ to $200 \mathrm{~cm}$. Unit adopts GPRS as the communication mode. The unit collects analog information of temperature, humidity, air pressure, light intensity within the working environment, and obligates interface to use for other analog monitoring. Positioning unit takes use of the typical GPS\&Wifi model combining with fingerprint positioning technology, which can realize accurate indoor positioning. Wheel control unit uses vector synthesis method to realize $360^{\circ}$ all-round freedom of movement. 


\section{Fingerprint positioning technology}

The fingerprint orientation method based on signal strength (RSS) is widely used in the process of WiFi positioning. The location of the mobile platform can be achieved by using the RSS measurement values to compare with fingerprint database stored value. ${ }^{[5]-[6]}$ Assumes that the RSS strength value of movement platform measured at some point in the indoor is $\left\{\overline{R S S_{1}}, \overline{R S S_{2}}, \ldots, \overline{R S S_{n}}\right\}$, then introduces a set of weighted coefficient $\left(\alpha_{1}, \alpha_{2} \ldots, \alpha_{n}\right)$, meet the conditions:

$\alpha_{1}+\alpha_{2}+\ldots+\alpha_{n}=0$

According to the formu-

las for strength calculation:

$R S S^{\prime}=\alpha_{1} \overline{R S S_{1}}+\alpha_{2} \overline{R S S_{2}}+\ldots+\alpha_{n} \overline{R S S_{n}}$

Assuming that the distance between mobile platform with a WiFi node conforms to the formula:

$R S S(l)=R S S\left(l_{0}\right)-10 \theta \log \left(\frac{l}{l_{0}}\right)+I$

Among them, $R S S(l)$ is measured signal strength, $R S S\left(l_{0}\right)$ is signal strength of the benchmark reference point, $l_{0}$ values $100 \mathrm{~cm}, \theta$ is the obstacle factor, which values range between 0 and $1, I$ is the mean zero Gaussian noise. By (2) (3), we can mark n wireless signal RSS values with reference to $\mathrm{n}$ measurement points.

$$
\begin{aligned}
& \left\{\overline{R S S_{1}^{1}}, \overline{R S S_{2}^{1}}, \ldots, \overline{R S S_{n}^{1}}\right\} \\
& \left\{\overline{R S S_{1}^{2}}, \overline{R S S_{2}^{2}}, \ldots, \overline{R S S_{n}^{2}}\right. \\
& \ldots \\
& \left\{\overline{R S S_{1}^{n}}, \overline{R S S_{2}^{n}}, \ldots, \overline{R S S_{n}^{n}}\right.
\end{aligned}
$$

Each group accords with a condition (1), so we can get $\mathrm{n}$ position coordinates.

$$
\left\{\begin{array}{ccc}
R S S_{1}^{\prime 1} & R S S_{2}^{\prime 1} & R S S_{n}^{\prime 1} \\
R S S_{1}^{\prime 2} & R S S_{2}^{\prime 2} & R S S_{n}^{\prime 2} \\
& & \\
R S S_{1}^{\prime n} & R S S_{2}^{\prime n} & R S S_{n}^{\prime n}
\end{array}\right\}
$$

Simulation results is as shown in figure 3. The blue nodes represent actual location information, the red nodes represent the location information by using positioning algorithm, the results can meet the requirements of practical application.

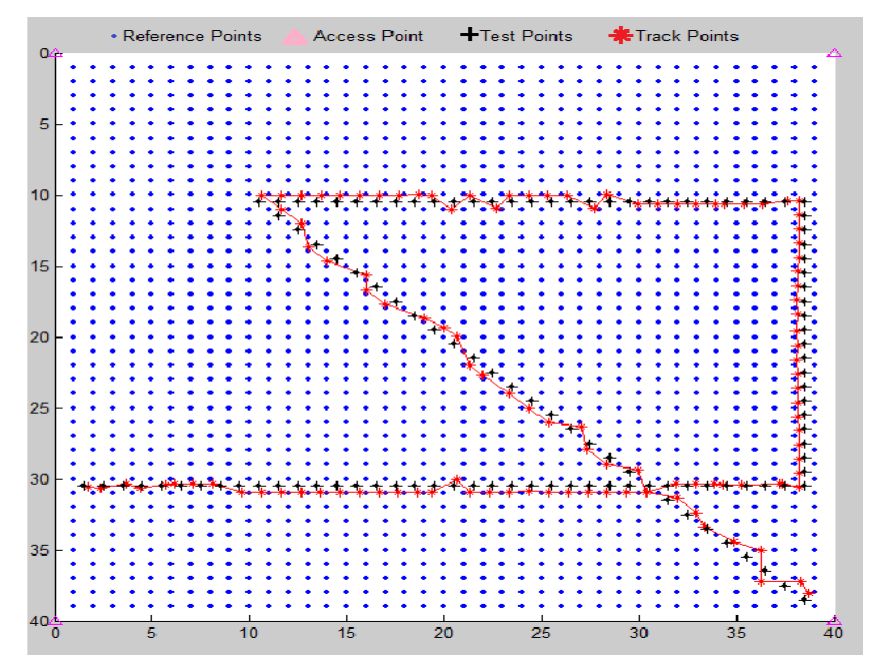

Figure 3. Indoor localization algorithm simulation diagram. 


\section{Omni-directional wheel structure}

Wheel structure is the key technology of mobile platform which directly affects the control complexity, flexibility, stability and the carrying capacity. This system designs an Omni-directional wheel according to the principle of cone lateral movement and the principle of differential mechanism. When the spherical surface of the taper contact with the ground, the force of contact point in the vertical direction should be unload by the movement around the axis of rotation. The force in the direction of the axis rotation is retained which is provides the movement of the wheel driving force. There is an interference point existed on the spherical top of the cone, and the reaction force on vertical plane also points to the axis of rotation which is unable to uninstall. So, it will be removed. ${ }^{[7][8]}$ Omnidirectional wheel structure is as shown in figure 4.

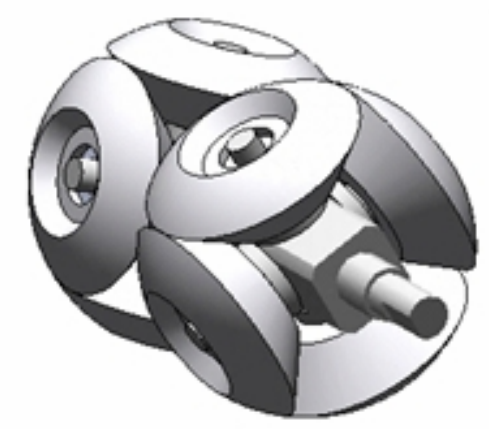

Figure 4. Omni-directional wheel structure.

There are three degrees of freedom on contact point: the drive freedom degree of rotation around the main shaft; the passive freedom degree around the cone of the rotating shaft; the passive rotation freedom degrees at the contact point. The three degrees of freedom fully ensure the realization of the comprehensive sporting function. Movement process is shown in figure 5.
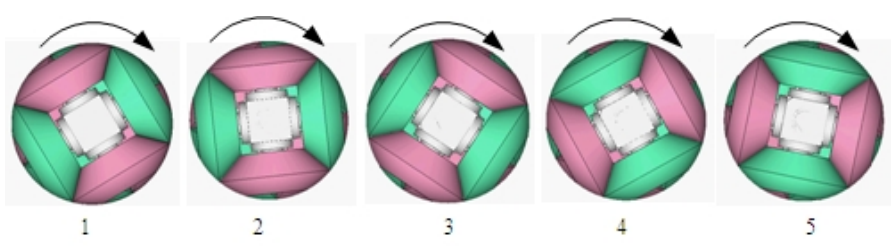

Figure 5.Omni-directional wheel movement process.

When the main drive shaft is in 1, 3, 5 positions shown in the graph, one group of the Omnidirectional wheel contact with the ground get to move. When the drive shaft in 2, 4 positions, another group of wheel get to move. The two groups of wheel alternately contact with the ground, and the contact contour line is still a complete circular curve.

To achieve the control of the mobile platform Omni-directional institutions, this paper established kinematics model which is as shown in figure 6.

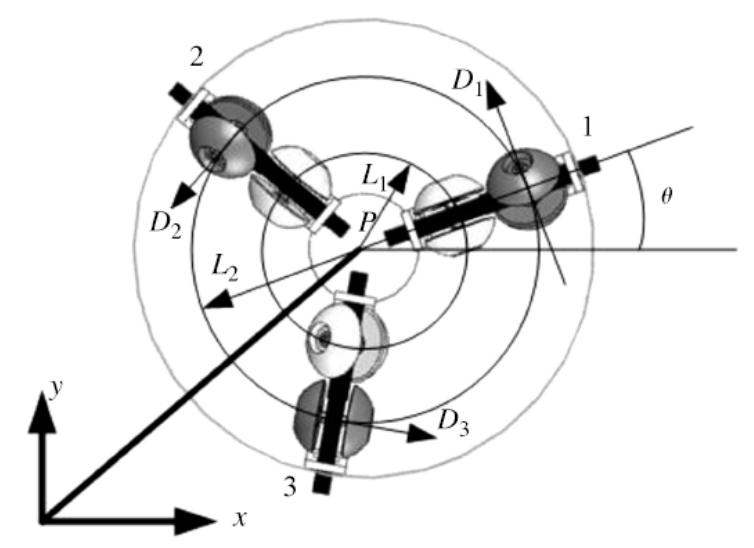

Figure 6. Omni-directional wheel kinematics model. 
The geometric constraint of complete movement driven model is:

$J \propto=R \phi$

The reverse movement mathematical model is:

$$
\frac{J \omega \alpha}{r}=\phi \delta
$$

Among them:

$$
\begin{aligned}
& J=\left\{\begin{array}{ccc}
-\sin \theta & \cos \theta & L \\
-\sin (\theta+2 \pi / 3) & \cos (\theta+2 \pi / 3) & L \\
-\sin (\theta+4 \pi / 3) & \cos (\theta+4 \pi / 3) & L
\end{array}\right\} \\
& \omega=\left[x_{p}, y_{p}, \theta\right]^{T}, \quad \phi=\left[\phi_{1}, \phi_{2}, \phi_{3}\right]^{T}, \quad \phi \quad \text { is rotation angles of three MY wheels. }
\end{aligned}
$$

\section{Conclusion}

To sum up, this system combines the Internet of things with robot technology, and implements a mobile platform design of high reliability, widely applicable. The platform takes advantage of the multiple sensor fusion and has realized a variety of function. At the same time, by using the fingerprint localization algorithm, it can greatly improve the indoor positioning accuracy which ensures that the mobile platform can be applied in special field.

Through experiment, this system can operate stable to meet requirements of the design. At the same time, this system can play an important roll in areas such as the Internet of things, robot applications, etc. It has a broad prospect for actual use.

\section{Acknowledgements}

Supported by Project of Shandong Province Higher Educational Science and Technology Program(NO. J14LN81)

\section{References}

[1] Tang Lili, Huang Wei, Wang Chunbo, Fang Gui, The Wifi Internet Car Design Based on CortexM3 Signal Chip. Radio Engineering, 2014 Vol.44 (4):p.58-61

[2] Zhao Jin, Zhu Sanchao, The design of intelligent obstacle avoidance car based on Arduino microcontroller, Automation and instrumentation, 2013(5), p.1-4.

[3] Jiang Kai, Gao Fan, Smart home system design based on the technology of Internet of things, Industrial control computer,2013,11:93-94

[4] Gao Shan, Fan Dehui, Jiang Kai*, Design of intelligent agricultural management system based on Internet of things technology, Advanced Materials Research. 2014(1008-1009): p.1479-1483

[5] Xiao Wendong, Song Zhipeng, Wifi indoor locating method based on linear encoding, Proceedings of the 33rd Chinese Control Conference (2014) , p482-485.

[6] P. Bahl, V. N. Padmanabhan, Radar: An In-Building RF based user Location and Tracking System, in proceedings of IEEE Infocom, 2000.pp.775-784

[7] Ye Changlong, Ma Shugen, Hui Li, An omni-directional mobile robot, China science, 2011 Vol.41(4):p.181-189.

[8] Ye Changlong, Li Huaiyong, Ma Shugen, Ni Huichao, The Kinematics Research of Omnidirectional mobile robot with MY wheel, The Robot, 2012 Vol.34(2): p.144-151. 